\title{
Efecto de variables operativas sobre la calidad de aplicación y la deriva en la pulverización de agroquímicos
}

\author{
Merani, V., Mur, M., Ramirez, F., Ponce, M., Guilino, F., Palancar, T. y Balbuena, R.
}

\begin{abstract}
RESUMEN
La pulverización con plaguicidas es la forma más difundida de control de plagas pero su uso es complejo, desde el punto de vista agronómico. Se realizó un ensayo a campo con el objeto de evaluar la incidencia de tres técnicas de aplicación de agroquímicos; en todas ellas se utilizó una pastilla abanico plano aire inducido, empleando distintas tasas de aplicación, velocidades de avance y distanciamientos entre pastillas quedando definidas tres técnicas: T1 (57 I ha-1, $18 \mathrm{~km} \mathrm{~h}^{-1}$ y 0,35 m), T2 (28,7 I ha-1, $18 \mathrm{~km} \mathrm{~h}^{-1}$ y 0,70 m) y T3 (57 I ha-1, $9 \mathrm{~km} \mathrm{~h}^{-1}$ y $0,70 \mathrm{~m}$ ). Para evaluar las técnicas se colocaron tarjetas hidrosensibles sobre el suelo y en columnas verticales, determinando la calidad de aplicación, la deriva de sedimentación y la exoderiva acumulada. Las tres técnicas fueron similares en calidad de aplicación alcanzando T1 una mayor cobertura. Para el factor exoderiva, T2 alcanzó diferencias significativas con respecto a las otras dos técnicas, por lo que altas velocidades de avance y distanciamientos entre picos y bajas tasas de aplicación aumentan la exoderiva. No hubo diferencias en deriva de sedimentación. Las variables operativas inciden sobre los riesgos ambientales independientemente del tamaño de gota producido.
\end{abstract}

Palabras clave: abanico plano; tasa de aplicación; exoderiva; bajo volumen.

Merani, V., Mur, M., Ramirez, F., Ponce, M., Guilino, F., Palancar, T. and Balbuena, R., 2019. Effects of operational variables on agrochemical spray application quality and drift. Agriscientia 36 (2): 45-55

\section{SUMMARY}

Pesticide spraying is the most widespread form of pest control. Spraying is a complex process, from the agronomic and environmental point of view. A field trial was carried out, to evaluate the effect of three agrochemical application 
techniques. In all of them, an air-induced flat fan was used, with different application rates, sprayer speeds and noozle spacing. Three techniques were defined: T1 (57 I ha-1, $18 \mathrm{~km} \mathrm{~h}^{-1}$ and 0,35 m), T2 (28,7 I ha-1, $18 \mathrm{~km} \mathrm{~h}^{-1}$ and 0,70 $\mathrm{m}$ ) and T3 (57 I ha-1, $9 \mathrm{~km} \mathrm{~h}^{-1}$ and 0,70 $\mathrm{m}$. To assess the techniques, watersensitive cards were placed on the ground in vertical columns, to measure the quality of application, sedimenting spray drift and cumulative airborne spray drift. The three techniques were similar in application quality but T1 reached a greater coverage. Regarding the airborne drift factor, T2 presented significant differences with respect to the other two techniques, which leads to the conclusion that higher speeds, larger noozle space and lower application rates increase airborne drift. There were no differences in sedimenting drift. Operational variables affect environmental risks regardless of the drop size produced

Keywords: flat fan; application rate; airborne drift; low volume.

Merani, V., Mur, M., Ramirez, F., Ponce, M.1, Guilino, F. y Palancar, T.: Facultad de Ciencias Agrarias y Forestales, Universidad Nacional de La Plata, 66 y 119, CP 1900, La Plata, Argentina. Correspondencia a: telmo@agro.unlp.edu.ar

\section{INTRODUCCIÓN}

Para mantener las adversidades bióticas por debajo del umbral económico, la práctica de mayor empleo se basa en el control químico y esto ha permitido el crecimiento de la producción agropecuaria. La Cámara de Sanidad Agropecuaria y Fertilizantes (CASAFE, 2014) informa que en los últimos 15 años el consumo de fitosanitarios en la Argentina pasó de 924,7 millones de dólares a 2381,2 millones en el año 2012.

La pulverización es la forma más difundida de aplicación de agroquímicos pero su uso es complejo, no solo desde el punto de vista agronómico, sino también por los riesgos de contaminación y para la salud humana (Onorato y Tesouro, 2004).

La mayoría de los pulverizadores agrícolas actuales utilizan pastillas hidráulicas para dosificar y atomizar el líquido. Las pastillas producen un amplio espectro en el tamaño de gota generado que van desde los 10 a los $1000 \mu m$ (Bouse, Kirk y Bode, 1990). Las gotas grandes tienen la ventaja de descender rápidamente y estar menos expuestas a la deriva por viento y por evaporación. Su principal desventaja es la reducción de deposición y adherencia sobre la superficie vegetal, pudiendo rebotar contra las hojas y caer al suelo en forma directa, o deslizarse y juntarse con otras gotas. A igualdad de volumen, es menor el número de impactos que se puede lograr con gotas grandes (Etiennot, 1993). Por lo contrario, las gotas pequeñas mejoran la retención por parte de las hojas y la cobertura, ofreciendo también una mejor penetración en el cultivo y la posibilidad de alcanzar la cara inferior de las hojas, tallos, etc. No obstante, las gotas más finas se evaporan más rápidamente, ya que tienen expuesta una mayor superficie reduciendo así su diámetro volumétrico mediano (DVM), y en consecuencia, la velocidad de sedimentación. Además, por su pequeño tamaño, son desviadas de su trayectoria por turbulencias del aire y térmicas (Sarubbi, 2010).

Para controlar las distintas plagas se requiere un número mínimo de impactos que varía con la plaga y con el producto. Márquez Delgado (2008) afirma que se requiere para la aplicación de herbicidas de preemergencia un mínimo de 20 a 30 gotas $\mathrm{cm}^{-2}$ con un DVM de 400-600 $\mu \mathrm{m}$, mientras que para herbicidas de contacto el número de impactos asciende de 30 a 40 gotas $\mathrm{cm}^{-2}$ con un DVM de 200-400 $\mu \mathrm{m}$. La Organización de las Naciones Unidas para la Alimentación y la Agricultura (FACO), citada por Leiva, Picapietra y Pergamino (2012), recomienda la misma cantidad para herbicidas, 20 a 30 gotas $\mathrm{cm}^{-2}$ para los insecticidas y fungicidas sistémicos o traslocables y 50 a 70 gotas $\mathrm{cm}^{-2}$ para los de contacto. Frola (2013) indica para la técnica de aplicación de bajo volumen 30 a 40 impactos $\mathrm{cm}^{-2}$.

Todas aquellas gotas que no lleguen hasta el blanco, o que aun habiéndolo alcanzado no permanezcan retenidas sobre él, constituyen una pérdida de producto que reduce la eficiencia de la 
aplicación. Este proceso se conoce genéricamente como deriva (Onorato y Tesouro, 2006). En este sentido, se debe considerar la fracción del fitoterápico que es transportada fuera del lote objeto del tratamiento, también llamada exoderiva (Nuyttens, De Schampheleire, Baetens y Sonck, 2007) y lo que cae dentro del lote, pero no sobre el objetivo, endoderiva (Villalba y Hetz, 2010). La exoderiva es un problema complejo por la gran cantidad de factores que la favorecen o promueven, y por la dificultad de cuantificar con precisión su magnitud y sus efectos ambientales. En general, se reconoce al tamaño de gota y a la velocidad del viento como factores determinantes del potencial de exoderiva (Lucero, 1998; Butler Ellis et al., 2002; Onorato y Tesouro, 2004; Al Heidary, Douzals, Sinfort y Vallet, 2014; Massaro, Rubén, García y Andrea, 2017). En cuanto a la determinación de la cantidad de producto exoderivado, Phillips y Miller (1999) encontraron que los valores disminuían con la altura de recolección. Mur et al. (2017) midiendo deriva en dos técnicas de pulverización, alto y bajo volumen, hallaron resultados similares.

Cavallo (2006) menciona que aproximadamente un $25 \%$ de la pulverización de plaguicidas da en el blanco; el resto afecta directamente a otros organismos hacia los cuales la aplicación no fue dirigida. Según Scramin et al. (2002) cerca del $32 \%$ del líquido aplicado es retenido por el objetivo, mientras que el $49 \%$ cae al suelo y el $19 \%$ va por el aire a áreas vecinas, afectando cultivos próximos y zonas habitadas. Martens (2012) menciona valores de eficiencia del 25 al $60 \%$, correspondiendo los menores registros a las aplicaciones sobre explotaciones arbóreas y los mayores a aquellas efectuadas en condiciones de barbecho y suelo desnudo. Chaim et al. (1999) encontraron que la deposición varía entre 24 y 41 $\%$ de acuerdo con el tamaño de las plantas de tomate, con pérdidas en el suelo entre 20 y $39 \%$ y pérdidas por deriva/evaporación entre 30 y $45 \%$.

Leiva (1995) agrupa los factores que inciden sobre la deriva en aquellos que corresponden al equipo, las técnicas de aplicación, las condiciones atmosféricas y las características de la aspersión. Las características de la aspersión afectan la deriva a través del tamaño de gotas. Indica que, trabajando con agua como dispersante, las gotas menores de $150 \mu \mathrm{m}$ están muy expuestas a la evaporación y deriva, y aquellas mayores de 350 $\mu \mathrm{m}$ no proveen una adecuada cobertura y caen generalmente al suelo arrastrando con ella al agroquímico. Considera que un adecuado balance se obtiene utilizando gotas entre $200-250 \mu \mathrm{m}$ de diámetro.
Una de las técnicas para reducir la exoderiva consiste en utilizar pastillas de inducción de aire que aumentan el tamaño de las gotas (Leiva, 2011). Este diseño permite que las gotas con aire se fragmenten cuando impactan, mejorando la cobertura del asperjado y la retención de las gotas. Domper, Mur y Balbuena (2015) cuantificaron la tasa de recuperación de producto por métodos fluorimétricos y procesos de digitalización de imágenes de tarjetas. Concluyeron que los programas de análisis sobreestiman la eficiencia de recuperación de las pastillas con aire inducido, debido a que la calculan a partir de la impresión de gotas cuyo volumen no es solamente líquido. Además, las gotas con aire se rompen con el impacto sobre el objetivo y producen gotas más pequeñas que son también contabilizadas en los cálculos. Butler Ellis et al. (2002) determinaron valores de $700-850 \mathrm{~kg} \mathrm{~m}^{-3}$ para la densidad de las gotas con aire inducido, lo que permite inferir una sobreestimación de al menos un 15 a $30 \%$ en el volumen de líquido recuperado.

Dentro de las principales variables operativas que inciden sobre la eficiencia de aplicación y el potencial de deriva, se encuentran aquellas que decide el operario de la máquina en cada aplicación. Se destacan la velocidad de trabajo, la altura de aplicación, la presión de trabajo del sistema y la distancia entre pastillas. Phillips y Miller (1999) mencionan que la exoderiva varía con la distancia entre pastillas, ya que se modifican los bordes expuestos de los abanicos contiguos, como también la mayor o menor porosidad en función de la superposición entre éstos.

Habitualmente, la tasa de aplicación -expresada en I ha-1- queda determinada por el caudal de la pastilla seleccionada, la velocidad de trabajo y la distancia entre pastillas. Para una pastilla dada, la tasa de aplicación también variará con la presión de trabajo seleccionada, la cual incide en el número y el tamaño de las gotas. Los operarios tienden a mantener una tasa de aplicación y modificar los demás parámetros de pulverización tratando de optimizar la eficiencia del equipo en cuanto a su capacidad de trabajo. Por lo tanto, si se aumenta la velocidad y se desea mantener la misma tasa de aplicación, se deberá aumentar el caudal de la pastilla o disminuir la distancia entre pastillas (Moltoni, Masiá y Moltoni, 2008; Valenzuela, 2016). El aumento de la velocidad podrá resolverse dentro de ciertos límites con un aumento de la presión de trabajo, lo cual implica una modificación del tamaño de gota producido. Estas dos últimas variables inciden fuertemente sobre el potencial de deriva. En un contexto en el que contratistas y productores buscan maximizar la capacidad operativa de sus 
equipos, es frecuente la reducción del volumen de aplicación sin una evaluación de sus efectos sobre la calidad de aplicación y el potencial de deriva.

Ferguson et al. (2016) encontraron mayor cobertura sobre el objetivo con mayores tasas de aplicación e indicaron que este factor tuvo más incidencia que el tamaño de gotas en el incremento de la cobertura. Legleiter y Johnson (2016) determinaron una mayor cobertura en la parte inferior del cultivo con mayores tasas de aplicación. Por el contrario, Domper et al. (2015) concluyeron que la mayor tasa de aplicación no garantiza lograr, por sí sola, un mayor número de impactos, cobertura y eficiencia. Frola (2013) considera que se pueden realizar aplicaciones eficientes con bajo volumen, 25 I ha- a 30 I ha-1 y gotas uniformes, entre 200 y $400 \mu \mathrm{m}$, de acuerdo al objetivo a controlar, sin grandes riesgos de afectar al cultivo adyacente. Sin embargo, advierte que uno de los mayores temores durante la aplicación es el aumento de la deriva. Indica que para minimizarla hay que evitar las gotas menores de 100 micrones, cuando las condiciones del viento lo requieran. Leiva et al. (2012), afirman que al bajar el volumen de pulverización, la incidencia del glifosato es mayor, ya que con menos agua se reduce la inactivación del glifosato.

Si bien se conocen los efectos generales de las variables operativas sobre la eficiencia y el potencial de deriva, son escasas las evaluaciones a campo de estas variables operativas actuando de manera concomitante. Por ejemplo, el aumento de la velocidad de trabajo conlleva una modificación del viento aparente y, como consecuencia, mayores riesgos de exoderiva. La velocidad de avance es considerada como responsable por el mayor movimiento lateral y vertical del botalón, pudiendo generarse a una mayor velocidad de trabajo menos impactos (Ooms, Ruter, Lebeau y Destain, 2003). También Sarubbi (2010) e Inostroza, Méndez y Ríos (2011) indican que la velocidad de avance influye en la calidad de aplicación.

Este trabajo pretende evaluar el efecto de tres técnicas de pulverización, conformadas por diferentes tasas, velocidades de avance y distanciamientos entre pastillas, sobre la eficiencia de aplicación y la deriva.

\section{MATERIALES Y MÉTODOS}

Los ensayos se realizaron en la Estación Experimental Julio Hirschhorn de la Facultad de Ciencias Agrarias y Forestales de la Universidad Nacional de La Plata ( $34^{\circ} 55^{\prime}$ S, $57^{\circ}$ 57' O). Para la aplicación se utilizó un tractor John Deere 6300 y una pulverizadora montada marca Hatsuta ${ }^{\circledR}$ con un tanque de 400 I y un botalón de 9,1 m de longitud, con 26 portapicos múltiples distanciados a $0,35 \mathrm{~m}$.

\section{Variables experimentales}

Se definieron tres técnicas de aplicación, en las cuales las variables experimentales fueron la tasa de aplicación, la velocidad de trabajo y el distanciamiento entre pastillas. Las demás variables intervinientes, altura de botalón, presión de trabajo y pastilla, fueron iguales para todas las técnicas.

Se establecieron dos tasas de aplicación que se denominaron de bajo y alto volumen, correspondiéndose con volúmenes de 28 । ha-1 y 57 I ha-1 respectivamente. Las pastillas se colocaron a una distancia de $0,35 \mathrm{~m}$ y de $0,70 \mathrm{~m}$; la velocidad de trabajo fue de $2,5 \mathrm{~m} \mathrm{~s}^{-1}$ y $5 \mathrm{~m} \mathrm{~s}^{-1}$ $\left(9 \mathrm{~km} \mathrm{~h}^{-1}\right.$ ó $\left.18 \mathrm{~km} \mathrm{~h}^{-1}\right)$. Con estas variables quedaron definidos tres tratamientos según la Tabla 1. Para todos los tratamientos se utilizaron pastillas de abanico plano Hypro® Al110015 de aire inducido (Al) conuna presión de $300 \mathrm{kPa}$ (3 bar) y una altura de pulverización de 0,75 m.

Se establecieron sectores de medición para la evaluación diferenciada de los efectos directos de la aplicación, a través de parámetros de calidad de aplicación, exoderiva y deriva de sedimentación. Para esto se separaron las mediciones en tres estaciones de muestreo con diferentes características, realizándose el pasaje en forma perpendicular a la dirección del viento predominante (Figura 2).

Para la evaluación de los efectos directos de la pulverización se trabajó sobre un rastrojo de soja con tres repeticiones y cuatro sectores de medición para cada repetición, en las cuales se colocaron

Tabla 1. Técnicas de aplicación con sus respectivas variables operativas

\begin{tabular}{lccccc}
\hline Referencia & $\begin{array}{c}\text { Tasa de aplicación } \\
\left(\mathrm{ha}^{-1}\right)\end{array}$ & $\begin{array}{c}\text { Velocidad de trabajo } \\
\left(\mathrm{km} \mathrm{h}^{-1}\right)\end{array}$ & $\begin{array}{c}\text { Distanciamiento entre } \\
\text { pastillas }(\mathrm{m})\end{array}$ & $\begin{array}{c}\text { Altura de } \\
\text { trabajo }(\mathrm{m})\end{array}$ & $\begin{array}{c}\text { Presión } \\
(\mathrm{bar})\end{array}$ \\
\hline T1 & 57,0 & 18 & 0,35 & 0,75 & 3 \\
T2 & 28,5 & 18 & 0,70 & 0,75 & 3 \\
T3 & 57,0 & 9 & 0,70 & 0,75 & 3 \\
\hline
\end{tabular}




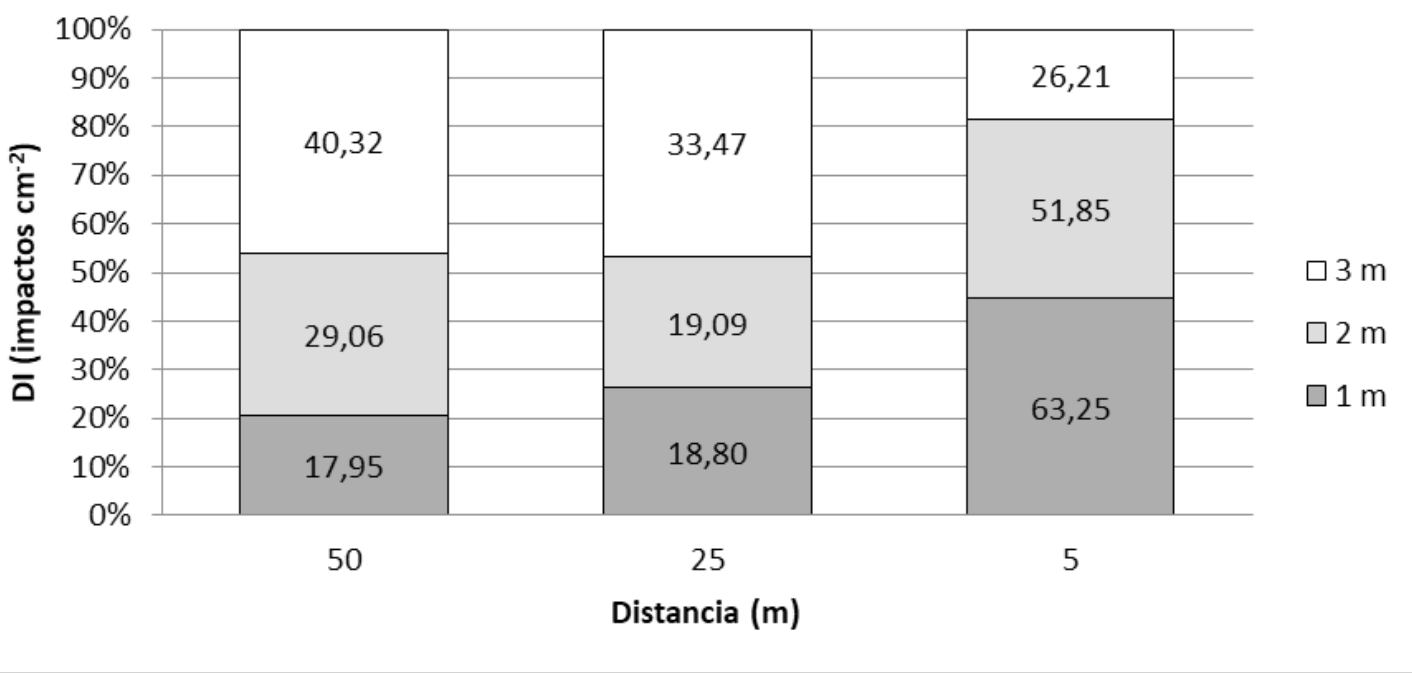

Figura 1. Exoderiva del tratamiento de bajo volumen (T2) en cada altura y distancia

dos tarjetas hidrosensibles ubicadas en forma transversal al avance de la máquina distanciadas a 1,04 m entre sí, en la parte media del botalón derecho, de manera tal de favorecer la evaluación de lugares coincidentes con la ubicación de las pastillas y de la zona de interacción de dos pastillas consecutivas.

El segundo sector de medición se colocó a 25 $\mathrm{m}$ del extremo del botalón en cada repetición, con una distribución de tarjetas igual a la de la primera estación, constituyendo cuatro sectores de dos tarjetas cada uno. Con los datos de esta estación se cuantificó la deriva de sedimentación, vinculada a la parte de la población de gotas que se deposita sobre el terreno, fuera del lugar directamente pulverizado por la máquina en cada pasaje, por desplazamiento lateral de éstas.

La tercera estación de muestreo para la medición de la exoderiva estuvo compuesta por tres torres de $3 \mathrm{~m}$ de altura ubicadas a 5,25 y 50 $\mathrm{m}$ del extremo del botalón, en cada repetición de cada tratamiento. En estas estaciones de muestreo se colocaron tarjetas de a pares a 1, 2 y $3 \mathrm{~m}$ de altura. Con los datos de esta estación se cuantificó la exoderiva acumulada para cada altura de medición. Posterior al pasaje correspondiente a cada repetición de cada tratamiento y distancia, las tarjetas fueron retiradas, calculándose la deriva acumulada por la sumatoria de los valores de densidad de impactos, eficiencia y cobertura. Las variables de caracterización de la población de gotas fueron promediadas para cada altura y distancia al extremo del botalón.

En cada aplicación se tomaron datos de temperatura, humedad relativa y velocidad del viento, proporcionados por una estación meteorológica portátil Kestrel 3500 DT $^{\circledR}$ (Tabla 2). Para la cuantificación de resultados se utilizaron tarjetas de papel sensible al agua, Syngenta ${ }^{\circledR}$ de $7,5 \mathrm{~cm} \times 2,5 \mathrm{~cm}$, las que posteriormente fueron digitalizadas por medio de un escáner Canon Slide $90^{\circledR}$ con una resolución de 1200 dpi. El procesamiento de las imágenes se realizó con el programa CIR $1.5^{\circledR}$ obteniendo número de impactos ( $\mathrm{n}^{\circ}$ de improntas $\mathrm{cm}^{-2}$ ) cantidad de gotas por unidad de superficie, diámetro volumétrico mediano (DV 0,5 o DVM) ( $\mu \mathrm{m})$, diámetro de gota que divide el volumen pulverizado a la mitad, diámetro numérico mediano (DNM) $(\mu \mathrm{m})$, diámetro de gota que divide el número de improntas halladas a la mitad, diámetro volumétrico 0,9 (DV 0,9) ( $\mu \mathrm{m})$ diámetro de gota por debajo del cual se encuentra

Tabla 2. Condiciones atmosféricas al momento de realizar el ensayo. $T^{\circ}$ : temperatura; HR: humedad relativa; Viento: velocidad del viento; P. atm: presión atmosférica.

\begin{tabular}{lcccccc}
\hline $\begin{array}{l}\text { Horario de } \\
\text { muestreo }\end{array}$ & T'C & HR \% & Presión $(\mathrm{atm})$ & $\begin{array}{c}\text { Viento max } \\
(\mathrm{km} / \mathrm{h})\end{array}$ & $\begin{array}{c}\text { Viento promedio } \\
(\mathrm{km} / \mathrm{h})\end{array}$ & delta T \\
\hline $12: 00 \mathrm{~h}$ & 26,0 & 64 & 1,011 & 18 & 14,6 & 5,5 \\
$14: 15 \mathrm{~h}$ & 28,2 & 54 & 1,008 & 15 & 13,0 & 7,1 \\
\hline
\end{tabular}


el $90 \%$ del volumen total del líquido recolectado, diámetro volumétrico $0,1(\mathrm{DV} 0,1)(\mu \mathrm{m})$, diámetro de gota por debajo del cual se encuentra el $10 \%$ del volumen total del líquido recolectado; eficiencia (TR)(\%), relación entre lo recogido en el objetivo y lo aplicado, área de cobertura (C) (\%) y porcentaje del área de la tarjeta manchada.

Se utilizó un diseño factorial, con tres repeticiones y ocho mediciones en cada repetición para los factores de calidad de aplicación y deriva de sedimentación, mientras que para exoderiva se utilizaron tres repeticiones y seis mediciones para cada tratamiento, distancia y altura de medición, 1, 2 y $3 \mathrm{~m}$ sobre el nivel del suelo. En los casos en que el ANOVA alcanzó significación estadística, se utilizó la prueba de LSD con un nivel de significación del $5 \%$. Para cobertura y densidad de impactos, el análisis de varianza se efectuó sobre los valores transformados de los datos por el logaritmo natural de la raíz cuadrada y el logaritmo natural respectivamente para alcanzar los criterios de homogeneidad y homocedasticidad de la varianza, expresándose los resultados a través de los valores reales obtenidos.

\section{RESULTADOSY DISCUSIÓN}

En la Tabla 3 puede observarse que los parámetros de calidad de aplicación han sido similares con las tres técnicas de aplicación para densidad de impactos, tasa de recuperación, DVM y $D_{0,9}$. Sin embargo, la cobertura, el $D V_{0,1}$ y el DNM han presentado valores significativamente inferiores en T2.

Los menores registros del $\mathrm{DV}_{0,1}$, como así también de DNM de T2 indicarían una mayor susceptibilidad que T1 y T3 a la exoderiva y a la evaporación (Leiva, 1995) ante condiciones atmosféricas desfavorables, por la existencia de un mayor número de gotas de menor diámetro (Lucero, 1998; Butler Ellis et al., 2002; Onorato y Tesouro, 2004; Al Heidary et al., 2014; Massaro et al., 2017), en coincidencia con lo informado por Sarubbi (2010). La ausencia de diferencias en el $\mathrm{DV}_{0,9}$ indica que la parte de la población de gotas de mayor tamaño no difiere y es poco afectada por las pérdidas de solución.
Los valores de DVM (en el orden de $300 \mu \mathrm{m}$ ) están dentro del rango recomendado por Frola (2013), Márquez Delgado (2008) y Leiva (1995).

Respecto a la cantidad de impactos lograda sobre el objetivo, las tres técnicas superan los valores mínimos recomendados para el control de malezas con herbicidas sistémicos indicados por Márquez Delgado (2008), Leiva et al. (2012) y Frola (2013), siendo la T1 la que presenta el mayor número de impactos, adjudicable a la mayor tasa de aplicación y mayor superposición entre abanicos de pulverización. La mayor velocidad de avance no generó una menor cantidad de impactos contradiciendo parcialmente a Ooms et al. (2003), Inostroza et al. (2011) y Sarubbi (2010), pero el mayor distanciamiento (T3) produjo menores registros y mayor variabilidad de los mismos.

Los mayores valores de cobertura fueron para la mayor tasa de aplicación de T1 pero solo alcanzaron diferencias significativas con T2, correspondiente al menor volumen ha-1 a la mayor velocidad de avance y distanciamiento más amplio entre pastillas, en concordancia con lo encontrado por Ferguson et al. (2016) y Legleiter y Johnson (2016). Los resultados apoyan lo hallado por Domper et al. (2015) para cada diseño de pastilla en relación a la ausencia de significancia del efecto de la velocidad, pese a que la variación de la velocidad en este caso fue mucho mayor.

En cuanto a la caracterización de la población de gotas sobre el objetivo de aplicación es posible observar que el DNM fue significativamente menor para T2 con respecto a T1, pero no con T3. El DVM, en cambio, fue similar para todas las alternativas pero T2 tuvo también los menores valores. En función de los resultados de estos parámetros la reducción de la cobertura para T2 puede explicarse por una densidad de impactos intermedia, constituida por un mayor número de gotas de menor tamaño y volumen. Estos resultados pueden ser en parte atribuidos a diferencias en el volumen de aplicación (Etiennot, 1993) que afectan, en conjunto con aspectos operativos (Leiva, 1995), a la población de gotas que llega al objeto de aplicación. En el tratamiento T2 se combinan además condiciones que predisponen en mayor medida a la exoderiva, como el mayor distanciamiento entre pastillas y la menor tasa de

Tabla 3. Parámetros del factor calidad de aplicación para las tres técnicas de aplicación (T1, T2 y T3).

\begin{tabular}{|c|c|c|c|c|c|c|c|c|c|c|c|c|c|c|}
\hline \multirow[b]{2}{*}{$\mathrm{T} 1$} & \multicolumn{2}{|c|}{ Cobertura (\%) } & \multicolumn{2}{|c|}{ Eficiencia (\%) } & \multicolumn{2}{|c|}{$\mathrm{N}^{\circ}$ Impactos $\left(\mathrm{cm}^{-2}\right)$} & \multicolumn{2}{|c|}{ DNM $(\mu \mathrm{m})$} & \multicolumn{2}{|c|}{$\mathrm{DVM}(\mu \mathrm{m})$} & \multicolumn{2}{|c|}{ DV0,1 ( $\mu \mathrm{m})$} & \multicolumn{2}{|c|}{ DV0,9 ( $\mu \mathrm{m})$} \\
\hline & 9,56 & $b$ & 66,23 & $\mathrm{a}$ & 219 & $A$ & 78,88 & $b$ & 298,29 & a & 160,18 & $b$ & 543,89 & a \\
\hline T2 & 5,26 & a & 57,15 & $\mathrm{a}$ & 157 & $A$ & 60,40 & a & 274,30 & a & 105,04 & a & 496,88 & a \\
\hline T3 & 7,39 & $a b$ & 52,79 & $a$ & 119 & $A$ & 70,11 & $a b$ & 316,59 & a & 164,30 & $b$ & 542,92 & a \\
\hline
\end{tabular}

Letras diferentes en la misma columna representan diferencias estadísticas significativas $(p<0,05)$ 
aplicación, que generan abanicos más porosos y menos superpuestos entre sí (Murphy, Miller y Parkin, 2000). Esto favorecería el desprendimiento de las gotas tanto de los bordes como del centro de los abanicos de pulverización al incrementarse el viento aparente como consecuencia de la mayor velocidad de avance.

En cuanto a la incidencia de los factores velocidad de avance y distancia entre pastillas, para un mismo volumen de aplicación, no existieron diferencias significativas entre T1 y T3. No obstante, los valores de T1 tendieron a una mayor densidad de impactos, cobertura y eficiencia, sin alcanzar diferencias significativas. El efecto del viento aparente, mayor en T1 que en T3, habría tenido menos incidencia que el distanciamiento entre pastillas en la pérdida de producto.

Los valores de eficiencia encontrados son superiores a los mencionados por Cavallo (2006) y Chaim et al. (1999), pero este tuvo en su ensayo condiciones de menor HR\% con similares velocidades y $\mathrm{T}^{\circ}$ y cercanos a los indicados por Martens (2012) quien tuvo condiciones de $T^{\circ}$ similares y velocidades del viento levemente menores, pero la HR\% también fue inferior y en valores similares a los de Chaim et al. (1999). También fueron mayores a los informados por Scramin et al. (2002) en el cultivo de algodón sobre el estrato superior, atribuible en parte a las características de las pastillas utilizadas.

Sin embargo, en los valores de eficiencia no se tiene en cuenta que el programa utilizado no contempla el efecto de "impresión" que produce la gota con aire sobre la tarjeta analizada (Domper et al., 2015). Si bien los resultados para los dos volúmenes de aplicación son altos en comparación a los ya citados, podrían atribuirse en primera instancia a dos factores que inciden en las mediciones. Por un lado, la impronta de la obtenida sobre el papel hidrosensible responde al volumen de líquido y aire que en su conjunto conforman la gota, y los cálculos se efectúan en función de una gota "llena" existiendo una sobreestimación de entre el 15 y $30 \%$ del volumen (Butler Ellis et al., 2002). Por otra parte, en las aplicaciones con este tipo de pastillas, el estallido de las "burbujas" por rotura en el momento del impacto y la consecuente generación de gotas de menor tamaño, tendría un efecto aditivo para el cálculo del volumen recuperado ya que éste sumaría a la impronta de una gota sobredimensionada, las producidas luego del golpe primario contra el objeto de aplicación.

T1 tuvo mayores valores de cobertura y eficiencia que T3 (no significativas). El mayor distanciamiento entre pastillas de T3 favorecería la pérdida de gotas por deriva (por estar los abanicos menos superpuestos y protegidos entre sí) disminuyendo las que llegan al blanco, afectando la eficiencia por un aumento de la deriva (Onorato y Tesouro, 2006). El efecto del viento aparente en donde T1 duplica a T3 habría tenido menos incidencia que el distanciamiento entre picos en la pérdida de producto.

La técnica de bajo volumen, a pesar de reducir la tasa de aplicación a la mitad, alcanza los requerimientos de densidad de impactos para el control de malezas, pero compromete la cobertura del objeto de aplicación (Valenzuela, 2016), contando como principal beneficio la reducción del consumo de agua, el aumento de la autonomía de la pulverizadora (Moltoni et al., 2008) y la disminución de la inactivación del producto con el agua (Leiva et al., 2012).

En la Tabla 4 se puede observar la cantidad de producto correspondiente a la exoderiva acumulada, recolectada en las torres de deriva, a 50,25 y $5 \mathrm{~m}$ del extremo del botalón T2, supera significativamente en densidad de impactos, cobertura y eficiencia a las otras dos técnicas. En el análisis de exoderiva, en forma inversa a la valoración de los efectos de la aplicación por debajo del botalón, los registros más altos indican una menor eficiencia y mayores riesgos ambientales de la aplicación. La densidad de impactos acumulada supera los valores indicados por Márquez Delgado (2008) para el control de malezas pudiendo afectar en consecuencia cultivos vecinos, cuando el pasaje se realiza cercano a éstos.

En la Figura1 se detallan los aportes de impactos registrados en T2 para cada altura de medición de exoderiva en relación a la distancia del pasaje del extremo del botalón. El 63,25 \% de los impactos por exoderiva a $1 \mathrm{~m}$ de altura, corresponden al pasaje del botalón a $5 \mathrm{~m}$ de las torres de medición, mientras que las pasadas a 25 y $50 \mathrm{~m}$ de distancia aportan aproximadamente 18 a $19 \%$ del total. En forma contraria, a medida que la distancia al extremo del botalón aumenta, se incrementa el porcentaje de aporte a 2 y $3 \mathrm{~m}$ de altura. Los resultados contradicen en parte las afirmaciones de Phillips y Miller (1999) y Mur et al. (2017) ya que la cantidad de producto recuperada, valorada a través de la eficiencia, no disminuye con la altura de recolección para todas las distancias de medición de la exoderiva sino que aumenta a 25 y 50 m del extremo del botalón.

En cuanto a la distribución de los impactos para cada distancia, los mayores registros a $5 \mathrm{~m}$ de distancia se encuentran a $2 \mathrm{~m}$ de altura (46\%) mientras que a $3 \mathrm{~m}$ se registra un 16,45\%. 
Tabla 4. Exoderiva acumulada de $5 \mathrm{~m}, 25 \mathrm{~m}$ y $50 \mathrm{~m}$ de distancia del extremo del botalón en las tres técnicas de aplicación (T1, T2 y T3). h1: $1 \mathrm{~m}$ de altura; h2: $2 \mathrm{~m}$ de altura; h3: $3 \mathrm{~m}$ de altura. Letras diferentes en la misma columna (dentro de cada altura) representan diferencias estadísticas significativas $(p<0,05)$ test $L S D$, valores entre paréntesis corresponden a desvió estándar.

\begin{tabular}{|c|c|c|c|c|c|c|c|c|c|c|c|c|c|c|}
\hline \multirow[b]{3}{*}{ T1 } & \multicolumn{14}{|c|}{ h1 } \\
\hline & \multicolumn{2}{|c|}{$\begin{array}{c}\text { Cobertura } \\
(\%)\end{array}$} & \multicolumn{2}{|c|}{$\begin{array}{c}\text { Eficiencia } \\
(\%)\end{array}$} & \multicolumn{2}{|c|}{$\begin{array}{l}\text { Impactos } \\
\left(\mathrm{cm}^{-2}\right)\end{array}$} & \multicolumn{2}{|c|}{$\begin{array}{l}\text { DNM } \\
(\mu \mathrm{m})\end{array}$} & \multicolumn{2}{|c|}{$\begin{array}{l}\text { DVM } \\
(\mu \mathrm{m})\end{array}$} & \multicolumn{2}{|c|}{$\begin{array}{l}\text { DV0,1 } \\
(\mu \mathrm{m})\end{array}$} & \multicolumn{2}{|c|}{$\begin{array}{l}\text { DV0,9 } \\
(\mu \mathrm{m})\end{array}$} \\
\hline & $\begin{array}{c}0,40 \\
(0,28)\end{array}$ & $\mathrm{a}$ & $\begin{array}{c}0,66 \\
(0,47)\end{array}$ & $a$ & $\begin{array}{c}49 \\
(34)\end{array}$ & a & $\begin{array}{l}46,58 \\
(2,16)\end{array}$ & $a$ & $\begin{array}{l}55,52 \\
(5,17)\end{array}$ & a & $\begin{array}{l}39,89 \\
(1,93)\end{array}$ & a & $\begin{array}{c}87,18 \\
(19,07)\end{array}$ & a \\
\hline T2 & $\begin{array}{c}0,85 \\
(0,07)\end{array}$ & a & $\begin{array}{c}1,98 \\
(0,02)\end{array}$ & $a$ & $\begin{array}{l}117 \\
(11)\end{array}$ & a & $\begin{array}{l}48,27 \\
(1,77)\end{array}$ & $a b$ & $\begin{array}{l}63,07 \\
(4,55)\end{array}$ & $a b$ & $\begin{array}{l}41,95 \\
(1,96)\end{array}$ & $a b$ & $\begin{array}{l}124,21 \\
(20,95)\end{array}$ & $b$ \\
\hline \multirow[t]{3}{*}{ T3 } & $\begin{array}{c}0,45 \\
(0,35) \\
\end{array}$ & a & $\begin{array}{c}1,45 \\
(0,85) \\
\end{array}$ & $a$ & $\begin{array}{c}43 \\
(20) \\
\end{array}$ & a & $\begin{array}{l}52,28 \\
(6,28) \\
\end{array}$ & $b$ & $\begin{array}{c}73,41 \\
(13,27) \\
\end{array}$ & $b$ & $\begin{array}{l}47,01 \\
(7,14) \\
\end{array}$ & b & $\begin{array}{r}105,60 \\
(16,37) \\
\end{array}$ & $a b$ \\
\hline & \multicolumn{14}{|c|}{ h2 } \\
\hline & \multicolumn{2}{|c|}{$\begin{array}{c}\text { Cobertura } \\
(\%)\end{array}$} & \multicolumn{2}{|c|}{$\begin{array}{c}\text { Eficiencia } \\
(\%)\end{array}$} & \multicolumn{2}{|c|}{$\begin{array}{l}\text { Impactos } \\
\left(\mathrm{cm}^{-2}\right)\end{array}$} & \multicolumn{2}{|c|}{$\begin{array}{l}\text { DNM } \\
(\mu \mathrm{m})\end{array}$} & \multicolumn{2}{|c|}{$\begin{array}{l}\text { DVM } \\
(\mu \mathrm{m})\end{array}$} & \multicolumn{2}{|c|}{$\begin{array}{l}\text { DV0,1 } \\
(\mu \mathrm{m})\end{array}$} & \multicolumn{2}{|c|}{$\begin{array}{c}\text { DV0,9 } \\
(\mu \mathrm{m})\end{array}$} \\
\hline T1 & $\begin{array}{c}0,20 \\
(0,00)\end{array}$ & a & $\begin{array}{c}0,46 \\
(0,11)\end{array}$ & a & $\begin{array}{l}25 \\
(6)\end{array}$ & a & $\begin{array}{l}46,45 \\
(4,61)\end{array}$ & $a$ & $\begin{array}{c}81,20 \\
(24,62)\end{array}$ & a & $\begin{array}{l}42,83 \\
(5,80)\end{array}$ & a & $\begin{array}{l}100,76 \\
(27,99)\end{array}$ & a \\
\hline T2 & $\begin{array}{c}1,60 \\
(0,28)\end{array}$ & $b$ & $\begin{array}{c}3,47 \\
(1,46)\end{array}$ & $a$ & $\begin{array}{l}175 \\
(39)\end{array}$ & b & $\begin{array}{l}52,36 \\
(6,38)\end{array}$ & $a$ & $\begin{array}{c}66,89 \\
(10,74)\end{array}$ & a & $\begin{array}{l}45,45 \\
(6,20)\end{array}$ & a & $\begin{array}{c}113,22 \\
(39,51)\end{array}$ & a \\
\hline \multirow[t]{3}{*}{ T3 } & $\begin{array}{c}0,20 \\
(0,28) \\
\end{array}$ & a & $\begin{array}{c}1,66 \\
(1,80) \\
\end{array}$ & $\mathrm{a}$ & $\begin{array}{c}28 \\
(26) \\
\end{array}$ & a & $\begin{array}{l}47,92 \\
(4,22) \\
\end{array}$ & $a$ & $\begin{array}{c}75,95 \\
(23,15) \\
\end{array}$ & a & $\begin{array}{l}44,22 \\
(6,49) \\
\end{array}$ & a & $\begin{array}{r}118,79 \\
(32,25) \\
\end{array}$ & a \\
\hline & \multicolumn{14}{|c|}{ h3 } \\
\hline & \multicolumn{2}{|c|}{$\begin{array}{c}\text { Cobertura } \\
(\%)\end{array}$} & \multicolumn{2}{|c|}{$\begin{array}{c}\text { Eficiencia } \\
(\%)\end{array}$} & \multicolumn{2}{|c|}{$\begin{array}{l}\text { Impactos } \\
\left(\mathrm{cm}^{-2}\right)\end{array}$} & \multicolumn{2}{|c|}{$\begin{array}{l}\text { DNM } \\
(\mu \mathrm{m})\end{array}$} & \multicolumn{2}{|c|}{$\begin{array}{l}\text { DVM } \\
(\mu \mathrm{m})\end{array}$} & \multicolumn{2}{|c|}{$\begin{array}{c}\text { DV0,1 } \\
(\mu \mathrm{m})\end{array}$} & \multicolumn{2}{|c|}{$\begin{array}{c}\text { DV0,9 } \\
(\mu \mathrm{m})\end{array}$} \\
\hline T1 & $\begin{array}{c}0,45 \\
(0,35)\end{array}$ & $a b$ & $\begin{array}{c}1,16 \\
(0,73)\end{array}$ & $a b$ & $\begin{array}{c}51 \\
(16)\end{array}$ & a & $\begin{array}{l}48,24 \\
(6,14)\end{array}$ & a & $\begin{array}{c}67,18 \\
(15,25)\end{array}$ & a & $\begin{array}{l}44,28 \\
(7,11)\end{array}$ & a & $\begin{array}{l}114,58 \\
(21,11)\end{array}$ & a \\
\hline T2 & $\begin{array}{c}0,95 \\
(0,07)\end{array}$ & b & $\begin{array}{c}2,36 \\
(0,03)\end{array}$ & b & $\begin{array}{l}124 \\
(18)\end{array}$ & b & $\begin{array}{l}48,24 \\
(2,64)\end{array}$ & a & $\begin{array}{l}66,27 \\
(8,62)\end{array}$ & a & $\begin{array}{l}42,47 \\
(3,75)\end{array}$ & a & $\begin{array}{l}107,33 \\
(27,07)\end{array}$ & a \\
\hline T3 & $\begin{array}{c}0,20 \\
(0,14) \\
\end{array}$ & a & $\begin{array}{c}0,71 \\
(0,07) \\
\end{array}$ & a & $\begin{array}{c}22 \\
(10) \\
\end{array}$ & a & $\begin{array}{l}49,42 \\
(5,92) \\
\end{array}$ & a & $\begin{array}{l}70,73 \\
(8,40) \\
\end{array}$ & a & $\begin{array}{l}44,25 \\
(4,93) \\
\end{array}$ & a & $\begin{array}{r}102,14 \\
(12,07) \\
\end{array}$ & a \\
\hline
\end{tabular}

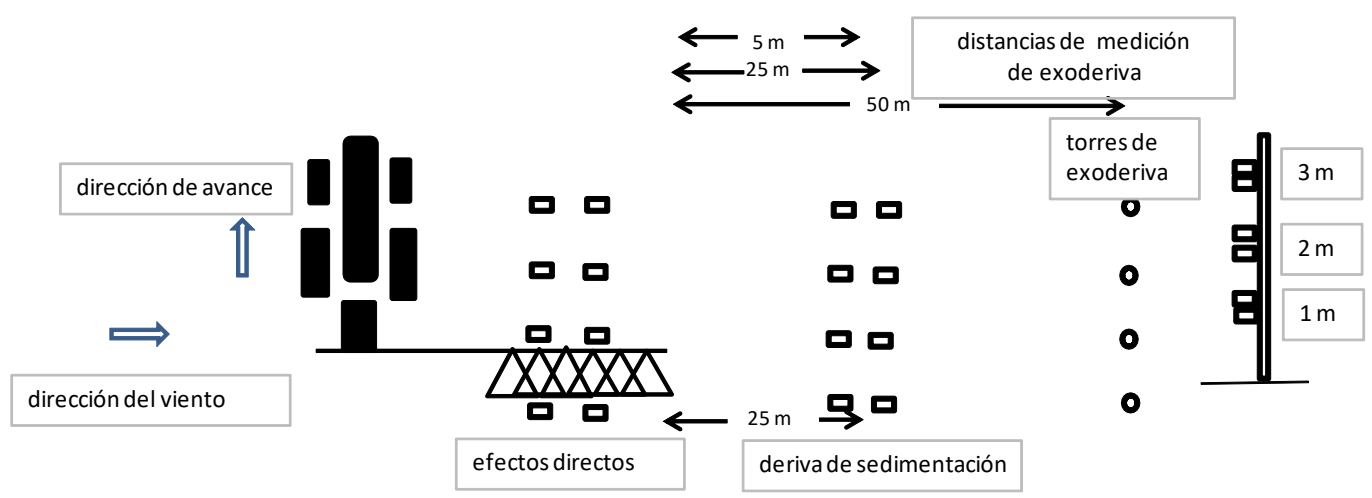

Figura 2. Disposición de las estaciones de muestreo para medición de: efectos directos (sobre el suelo, bajo el botalón), deriva de sedimentación (sobre el suelo a $25 \mathrm{~m}$ del extremo del botalón) y exoderiva (torres con 3 alturas a $5 \mathrm{~m}, 25 \mathrm{~m}$ y $50 \mathrm{~m}$ del extremo del botalón).

La reducción en el número de impactos registrada para 25 y $50 \mathrm{~m}$ con respecto a $5 \mathrm{~m}$ puede ser atribuida en parte a los procesos de evaporación durante el trayecto recorrido, como también a los procesos de elevación de la población de gotas exoderivadas por acción del viento. La ausencia de interacción entre alturas de medición y distancias al extremo del botalón indica que todos los tratamientos tuvieron similar comportamiento, pero con valores significativamente menores con respecto a T2

Las características de la población de las gotas exoderivadas son similares para las distintas 
técnicas de aplicación de manera independiente de las diferentes variables operativas. Las gotas tienen un DVM de 55 a $75 \mu \mathrm{m}$, se encuentran en la categoría muy fina (American Society of Agricultural Engineers, ASAE, 2009) y son además muy uniformes, estando expuestas por su pequeño tamaño a ser desviadas de su trayectoria por turbulencias del aire y térmicas (Sarubbi, 2010). El tamaño de gotas presentó pequeñas variaciones entre las distintas alturas de medición y distancias del pasaje de la pulverizadora a las torres de deriva, y coincide con lo citado por Leiva (1995) quien afirma que las gotas más propensas a derivarse son las de menos de $150 \mu \mathrm{m}$. T3 tuvo valores mayores de tamaño de gota en DVM, $\mathrm{DV}_{0,1}$ y DNM que T1 en la menor altura de evaluación de la exoderiva.

Si se comparan las Tablas 3 y 4 para densidad de impactos puede apreciarse que en T1 y T3 la relación de gotas halladas en exoderiva con las gotas encontradas en el objetivo fue de aproximadamente un $9 \%$ mientras que para la T2 esta relación fue de $21 \%$ por lo tanto con la técnica T2 ha aumentado la proporción de gotas derivadas.

Teniendo en cuenta la cantidad de impactos determinados con el menor volumen y la mayor velocidad, el riesgo potencial de exoderiva aumenta para la técnica de bajo volumen. Debe tenerse en cuenta además que la solución exoderivada con bajos volúmenes sería de mayor potencial de riesgo, por concentración del producto y por menor inactivación éste (Leiva et al., 2012).

Los resultados de mayor exoderiva para T2 resultan concordantes con el menor $\mathrm{DV}_{0,1}$ (Sarubbi, 2010) encontrado en su población de gotas (Tabla2).

En la técnica T3 se observó que la mayor cantidad de impactos disminuyó con la altura de recolección, en coincidencia con lo reportado por Phillips y Miller (1999) y Mur et al .(2017), pero no ocurrió lo mismo en T1 ni T2. Las ráfagas de viento existentes en el momento del ensayo podrían explicar estas anomalías y diferencias entre los tratamientos.
Es probable que con pastillas convencionales, los mismos tratamientos dieran mayores valores de exoderiva y las diferencias hubiesen sido más acentuadas. El uso de pastillas de baja deriva podría haber atemperado las diferencias entre las técnicas (Leiva, 2011)

En la Tabla 5 pueden verse los parámetros determinados para el factor deriva de sedimentación. Sólo se observan diferencias estadísticas en el DVM con gotas de mayor tamaño en T2 con respecto a T1. En el resto de los parámetros no hay diferencias, pero se aprecia una menor densidad de impactos en T2, ya que el conjunto de gotas derivadas es similar en tamaño a las encontradas para exoderiva.

El tamaño de gota permite clasificarlas dentro del rango mencionado por Leiva (1995) de gotas propensas a la evaporación y a ser desplazadas por el viento, por lo que es de esperar que dichas gotas sean encontradas fuera del objetivo. Además, el menor tamaño de gota hace que éstas se evaporen más rápidamente, ya que tienen expuesta una mayor superficie reduciendo así su DVM y en consecuencia la velocidad de sedimentación (Sarubbi, 2010). El DVM de la población de gotas confirma lo citado por Frola (2013), en cuanto a que se deben evitar las gotas menores a 100 micrones ante condiciones ventosas.

El número de impactos encontrados implica que con las tres técnicas sería factible que la exoderiva pueda afectar especies vegetales tanto con productos sistémicos como de contacto, que estuviesen en las zonas aledañas al lote tratado (Márquez Delgado, 2008).

\section{CONCLUSIONES}

Aun con bajos volúmenes y altas velocidades de aplicación, se alcanza el número de impactos necesario para el control de malezas con herbicidas sistémicos.

Las técnicas de aplicación con bajo volumen favorecen los procesos de exoderiva.

Tabla 5. Parámetros del factor deriva de sedimentación para las tres técnicas de aplicación (T1, T2 y T3). Letras diferentes en la misma columna representan diferencias estadísticas significativas $(p<0,05)$ test LSD

\begin{tabular}{|c|c|c|c|c|c|c|c|c|c|c|c|c|c|c|}
\hline \multirow[b]{2}{*}{$\mathrm{T} 1$} & \multicolumn{2}{|c|}{ Cobertura (\%) } & \multicolumn{2}{|c|}{$\begin{array}{c}\text { Eficiencia } \\
(\%)\end{array}$} & \multicolumn{2}{|c|}{$\begin{array}{c}\mathrm{N}^{\circ} \text { Impactos } \\
\left(\mathrm{cm}^{-2}\right)\end{array}$} & \multicolumn{2}{|c|}{ DNM ( $\mu \mathrm{m})$} & \multicolumn{2}{|c|}{$\begin{array}{l}\text { DVM } \\
(\mu \mathrm{m})\end{array}$} & \multicolumn{2}{|c|}{$\begin{array}{l}\text { DVO } 1 \\
(\mu \mathrm{m})\end{array}$} & \multicolumn{2}{|c|}{$\begin{array}{c}\text { DV0,9 } \\
(\mu \mathrm{m})\end{array}$} \\
\hline & 0,65 & $a$ & 1,48 & a & 91 & $a$ & 51,01 & a & 61,07 & $\mathrm{a}$ & 43,66 & $a$ & 91,28 & a \\
\hline $\mathrm{T} 2$ & 0,45 & a & 2,84 & a & 59 & a & 53,89 & $a$ & 75,81 & $b$ & 48,67 & $\mathrm{a}$ & 186,2 & a \\
\hline T3 & 0,70 & a & 1,90 & a & 80 & a & 52,38 & a & 72,84 & $a b$ & 46,84 & $\mathrm{a}$ & 180,93 & a \\
\hline
\end{tabular}


El uso de pastillas con inducción de aire no elimina la pérdida de producto por exoderiva si las variables de velocidad de avance y distancia entre pastillas incrementan los efectos del viento sobre el abanico de pulverización.

\section{BIBLIOGRAFÍA}

Al Heidary, M., Douzals, J. P., Sinfort, C. y Vallet, A. (2014). Influence of spray characteristics on potential spray drift of field crop sprayers: A literature review. Crop Protection, 63, 120-130.

American Society of Agricultural Engineers (ASAE) 2009. S572.1. Spray Nozzle Classification By Droplet Spectra.

Bouse, L. F., Kirk, I. W. y Bode, L. E. (1990). Effect of spray mixture on droplet size. Transactions of the ASAE, 33(3), 783-788.

Butler Ellis, M., Swan, T., Miller, P. C. H., Waddelow, S., Bradley, A. y Tuck, C. R. (2002). PM-power and machinery: design factors affecting spray characteristics and drift performance of air induction nozzles. Biosystems Engineering, 82(3), 289-296.

Cámara de Sanidad Agropecuaria y Fertilizantes (CASAFE) (2014). Mercado Argentino de Productos Fitosanitarios 2012. Recuperado dehttp://www.casafe. org/publicaciones/estadisticas/

Cavallo, A. (2006). Plaguicidas: qué son y cómo usarlos. Universidad Nacional de Córdoba, Córdoba, Argentina: Editorial SIMA.

Chaim, A., de Castro, V. L. S. S., Corrales, F. M., Galvão, J. A. H., Cabral, O. M. R. y Nicolella, G. (1999). Método para monitorar perdas na aplicação de agrotóxicos na cultura de tomate. Pesquisa Agropecuária Brasileira, 34(5), 741-747.

Domper, G. N., Mur, M. y Balbuena, R. H. (2015). Eficiencia de aplicación de pastillas de pulverización con inducción de aire en el cultivo de soja. Revista de la Facultad de Agronomía, La Plata, 113(2), 202-210.

Etiennot, A. E. (1993). Pulverizaciones terrestres. Segundo Congreso Nacional de Siembra Directa. Septiembre 1993. Huerta Grande, Córdoba, Argentina. 163-173.

Ferguson, J. C., Chechetto, R. G., Hewitt, A. J., Chauhan, B. S., Adkins, S. W., Kruger, G. R. y O'Donnell, C. C. (2016). Assessing the deposition and canopy penetration of nozzles with different spray qualities in an oat (Avena sativa L.) canopy. Crop Protection, 81, 14-19.

Frola, E. (2013). Manejar tamaño, número y distribución de impactos para lograr aplicaciones de calidad. Todo Agro. Recuperado de: http://www.todoagro.com. ar/noticias/nota. $a s p ?$ nid=25566.
Inostroza, J., Méndez P. y Ríos, P. (2011). Manual de campo. Uso de equipos pulverizadores. Boletín INIA N 25, Chile 76 p. Recuperado de http://static.elmercurio. cl/Documentos/Campo/2013/02/07/20130207174510. pdf

Legleiter, T. R. y Johnson, W. G. (2016). Herbicide coverage in narrow row soybean as influenced by spray nozzle design and carrier volume. Crop Protection, 83, 1-8.

Leiva, A. P. D., Picapietra, G. y Pergamino, G. P. V. I. (2012). Compatibilidad para mezclas de tanque de tres herbicidas utilizados en barbecho químico. Grupo Protección Vegetal-INTA Pergamino. Argentina., 11 $\mathrm{pp.}$

Leiva, P. D. (1995). Manejo de la deriva en la aplicación de agroquímicos. Carpeta de Producción Vegetal. INTA, EEA Pergamino, SERIE: Generalidades, 15 (139), 6.

Lucero, E. M. (1998). Análisis comparativo de la deriva producida por pastillas pulverizadoras hidráulicas e hidroneumáticas, tipo abanico plano. Cátedra de Maquinaria Agrícola. Trabajo de intensificación. Maquinaria Agrícola. UBA. 9 pp.

Márquez Delgado, L. (2008). Buenas prácticas agrícolas en la aplicación de fitosanitarios. Ministerio de Medio Ambiente y Medio Rural y Marino. Secretaría General Técnica Centro de Publicaciones. NIPO: 770-08-1583. 33.

Martens, F. (2012). Guía para el uso adecuado de plaguicidas y la correcta disposición de sus envases. Boletín de divulgación INTA, 41: 26. Tandil: Publicaciones Regionales INTA

Massaro, A., Rubén, A., García, A. y Andrea, V. (2017). Evaluación de la deriva en pulverización aérea y terrestre con plaguicidas en situación de barbecho. Boletin INTA Oliveros. Recuperado de https://inta. gob.ar/sites/default/files/inta-ensayo-deriva-aerea-yterrestre.pdf.

Moltoni, L., Masiá, G. y Moltoni, A. (2008). Cost and income analysis of agricultural labors: the role of providers of agricultural machinery services in argentina. In Central theme, technology for all: sharing the knowledge for development. Proceedings of the International Conference of Agricultural Engineering, XXXVII Brazilian Congress of Agricultural Engineering, International Livestock Environment SymposiumILES VIII, Iguassu Falls City, Brazil, 31st August to 4th September, 2008. International Commission of Agricultural Engineering (CIGR), Institut fur Landtechnik.

Mur, M., Ponce, M. J., Vázquez, J. M., Guilino, F., Merani, V., Palancar, T. y Balbuena, R. H. (2017). Control de malezas en pre siembra: alternativas tecnológicas y riesgos ambientales de la aplicación. Revista de la 
Facultad de Agronomía, Universidad Nacional de La Plata Vol 116 (2): 267-277

Murphy, S. D., Miller, P. C. H. y Parkin, C. S. (2000). The effect of boom section and nozzle configuration on the risk of spray drift. Journal of agricultural engineering research, 75(2), 127-137.

Nuyttens, D., De Schampheleire, M., Baetens, K. y Sonck, B. (2007). The influence of operator-controlled variables on spray drift from field crop sprayers. Transactions of the ASABE, 50 (4), 1129-1140.

Onorato, A. y Tesouro M. O. (2006). Pulverizaciones Agrícolas Terrestres. Buenos Aires, Argentina: Ed. INTA.

Onorato, A. A., y Tesouro, M. O. (2004). Desempeño antideriva de una boquilla de pulverización agrícola de cono hueco inducida por aire. RIA. Revista de Investigaciones Agropecuarias, 33 (2).

Ooms, D., Ruter, R., Lebeau, F. y Destain, M. F. (2003). Impact of the horizontal movements of a sprayer boom on the longitudinal spray distribution in field conditions. Crop protection, 22(6), 813-820.

Phillips, J. C. y Miller, P. C. H. (1999). Field and wind tunnel measurements of the airborne spray volume downwind of single flat-fan nozzles. Journal of Agricultural Engineering Research, 72(2), 161-170.

Sarubbi, C. A. (2010). Tecnología de aplicación de productos fitosanitarios en equipos pulverizadores terrestres (No. 632.94)p. 68-69. Buenos Aires, Argentina: Editorial Facultad de Agronomía. Universidad de Buenos Aires.

Scramin, S., Chaim, A., Pessoa, M. C. P. Y., Ferracini, V. L., Pavan, L. A. y Alvarenga, N. (2002). Avaliação de bicos de pulverização de agrotóxicos na cultura do algodão. Pesticidas: Revista Ecotoxicologia e Meio Ambiente, 12, 43-50.

Valenzuela, L. (2016). Pulverización con máquinas de botalón y bajo volumen. Tesis de grado, Facultad de Ciencias Agrarias y Forestales, Universidad Nacional de La Plata, La Plata, Buenos Aires, Argentina. Recuperado de: http://sedici.unlp.edu.ar/ handle/10915/53023.

Villalba, J. y Hetz, E. (2010). Deriva de productos agroquímicos - Efecto de las condiciones ambientales. Tecnología de aplicación de agroquímicos. Argentina: Área de comunicaciones del INTA Alto Valle, 45-54. 